\title{
The Optimization and Design of Magnetic Structure of MRF Damper
}

\author{
GUO Zhijun ${ }^{1, a}$, WEI Fei ${ }^{1, b}$ \\ ${ }^{1}$ Vehicle \& Transportation Engineering Institute, Henan University of Science and Technology, \\ LUOyang, Henan,China, 471003 \\ aemail:gzhi1970@163.com, bemail:284684633@qq.com
}

\begin{abstract}
Keywords: MRF damper; magnetic structure; optimal design; finite element analysis
Abstract. Based on the classical magnetic structure of the vehicle MRF damper, this paper mainly focuses on the influence of magnetic circuit structure and key parameters on response time of MRF damper, and put forward the optimization method of magnetic circuit structure, which adopted the double stage magnetic circuit and optimized the calculation of key parameter like working cylinder, to ensure that the magnetic circuit design meets performance requirements and the response time of magnetic circuit is the shortest. The finite element software ANSYS was used to simulation analysis for magnetic circuit structure. The result of the simulation analysis shows that the performance of optimized MRF damper is better, for the magnetic field distribution is more reasonable, the magnetic flux density increases obviously, and the response time is reduced effectively.
\end{abstract}

\section{Introduction}

Magnetorheological fluid (MRF) is a kind of intelligent material controlled by magnetic field with rheological property which consist of carrier fluid, paramagnetic particle or ferromagnetic particles and additive dispersed in the carrier fluid. Under the action of an external magnetic field, the liquid viscosity can change in millisecond time, and the shear stress can be effectively controlled by the change of the magnetic field [1]. Magnetorheological fluid is widely used in the industrial field, because it has the characteristics like short response time, wide dynamic range, low manufacturing cost, simple structure and no hydraulic valve vibration shock and so on, making it shows a good application prospect in the automotive field.

MRF damper that use magnetorheological fluid as working medium, is a new intelligent vibration damper. Making the internal magnetic field strength shock absorber damper current change by controlling the electromagnetic coil size, to change the yield stress of magnetorheological fluid is its working principle, so that the damping force can be adjusted continuously. Duo to the characteristics of magnetorheological fluid, the design of magnetic circuit should not only meet the requirements of the damping force work, but need to provide the corresponding magnetic field strength at the same time, to avoid magnetic circuit saturation, otherwise the magnetic field strength and the magnetic field intensity will be insufficient [2]. All the structural parameters in the magnetic circuit will have an impact on the overall performance of the damper. Therefore, choosing the reasonable magnetic circuit structure and parameters are important to design the damper [3].

Besides the damping force and adjusting range of MRF damper, the response time is also an important performance index [4]. The research at home and abroad, mainly focus on the control strategy and damping force model analysis, however the research on the response time of the absorber is much less. In this paper, the influence of response time on the performance of MRF damper is studied [5]. Combined with different magnetic circuit structure and key parameters, and considering the magnetic properties of the material, the structure of the magnetic circuit is optimized. By using ANSYS finite element software, the magnetic field characteristics of MRF damper are simulated and analyzed. Compared the magnetic field distribution before and after optimizing, the reasonability of the magnetic circuit optimization design can be verified.2. Magnetic circuit design of MRF damper. 


\section{Basic Theory of Magnetic Circuit}

The structure of a MRF damper in a car is shown in Fig. 1. The working parts are made up of piston rod, piston, coil, cylinder, working gap and magnetorheological fluid.

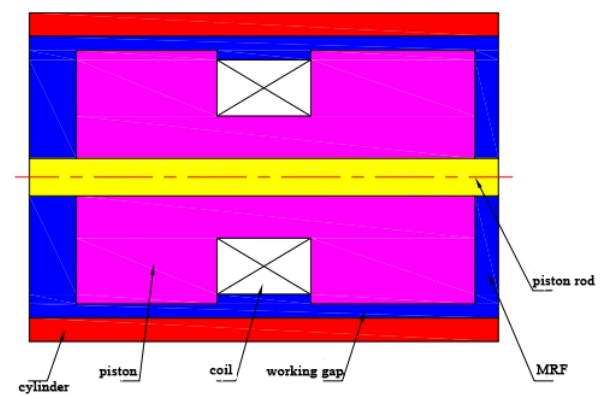

Fig.1 Structure diagram of MRF damper

Through analyzing the Ohm's law, Gauss law and Ampere law among magnetic flux of the magnetic circuit, the magnetic field intensity and excitation current, [6] the basic parameters of magnetic circuit have reluctance $\mathrm{Rm}$, the magnetic flux ${ }^{\emptyset}$, magnetic potential $\mathrm{F}$, and the relationship as follows:

$$
\mathrm{F}=\mathrm{Rm}^{\curvearrowleft} \text {. }
$$

Magnetic potential can also be expressed as:

$\mathrm{F}=\mathrm{NI}$.

Among them, $\mathrm{N}$ is coil turn, and $\mathrm{I}$ is exciting current.The flux is up to:

$$
\emptyset=\int, b d \cdot s \text {. }
$$

Reluctance Rm can be represented as:

$$
R_{m=n s} \stackrel{b}{\text { s. }}
$$

Among them, $\mathrm{S}$ is the magnetic circuit area, $\mathrm{b}$ is the magnetic circuit average length, and $\mu$ is relative permeability.

From the Gauss law of magnetic field, the total magnetic flux through any magnetic surface $S$ must be zero, MRF damper, magnetic core, the edge of the piston, damping channel and the cylinder to form a closed loop, through the flux of each part are the same.

$$
\phi_{\text {total }}=\phi_{1}=\phi_{2}=\phi_{3}=\phi_{4} .
$$

\section{Calculation of Reluctance}

The magnetic circuit diagram is shown in Fig. 1 [7]. Where L1 is the length of the piston, L2 is the damping channel length, $\mathrm{r} 1$ is the piston rod through hole (to lead wire) radius [8], $\mathrm{r} 3$ is the piston radius, $\mathrm{r} 4$ is the outer radius of piston sleeve, $\mathrm{g}$ is the working gap width of magnetorheological fluid, and $t$ is the working cylinder thickness.

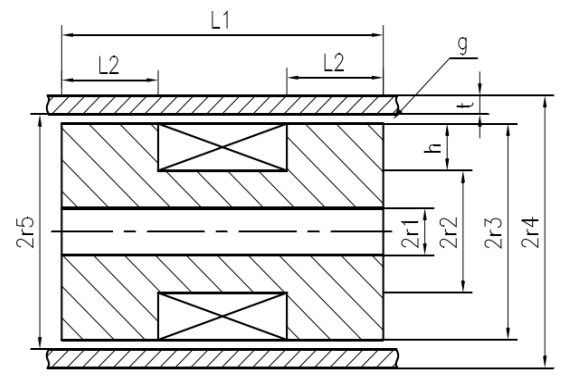

Fig. 2 Magnetic circuit structure of MRF damper 
According to the structural characteristics of the absorber, the the magnetic circuit can be divided into 4 parts, A1, A2, A3 and A4 which is working slot area. Based on the equivalent principle, the reluctance of each region is calculated, then the whole magnetic circuit is equivalent to the series reluctance structure, thus the total reluctance of the magnetic circuit can be obtained.

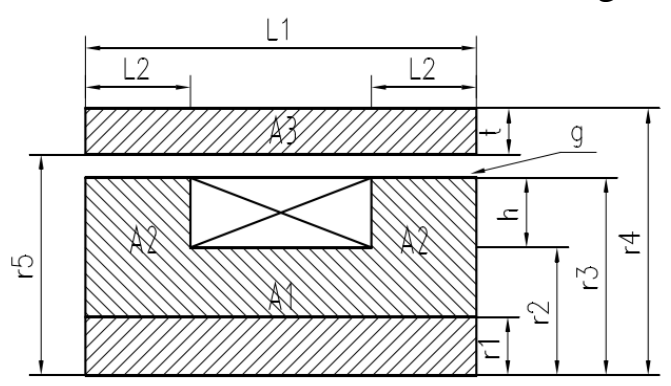

Fig. 3 The calculation diagram of magnetic circuit reluctance

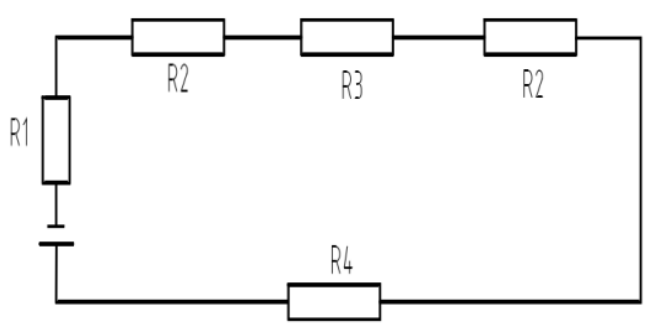

Fig. 4 Equivalent series reluctance structure

In Fig. 4, R1 is the reluctance of A1, R2 is the reluctance of A2, R3 is the reluctance of A3, and $\mathrm{R} 4$ is the reluctance of the magnetorheological fluid working gap.

The reluctance of the A1 region is:

$$
R_{1}=\frac{L_{1}-L_{2}}{\mu \pi \mu_{r p}\left(r_{2}^{2}-r_{1}^{2}\right)} \text {. }
$$

The reluctance of the $\mathrm{A} 2$ region is:

$$
R_{2}=\frac{r_{2}-r_{1}}{2 \mu_{0} \pi \mu_{r p} r_{2} L_{2}} .
$$

The reluctance of the $\mathrm{A} 3$ region is:

$$
R_{3}=\frac{L_{1}-L_{2}}{\mu_{0} \pi \mu_{r h}\left(2 r_{4}^{2}-r_{5}^{2}\right)} \text {. }
$$

The reluctance of the $\mathrm{A} 4$ region is:

$$
R_{4}=\frac{\ln \left(1+\frac{g}{r_{3}}\right)}{2 \mu_{0} \pi L_{2} \mu_{m R}} .
$$

The total reluctance of magnetic circuit is:

$$
R_{\text {total }}=R_{1}+R_{2}+R_{3}+R_{4} \text {. }
$$

In formula, $\mu_{0} \mu_{r p} \mu_{r h} \mu_{m R}$ are vacuum permeability, relative permeability of working cylinder, piston and magnetorheological fluid.The total magnetic potential is:

$\mathrm{F}=0 . R_{\text {total }}$. 
According to the total magnetic potential F, and the maximum excitation current Imax of the power and heat dissipation condition of the damper, the required coil turns can be determined:

$$
N=F / I_{\max }
$$

\section{Calculation of Response Time of Magnetic Coil}

According to Faraday law of electromagnetic induction, the response time constant for magnetic coil.

$\tau=K / R$.

In formula, $\mathrm{K}$ is the magnetic induction coefficient, and $\mathrm{R}$ is reluctance.

$$
R=\frac{b}{\mu S}
$$

In formula, $b$ is the magnetic circuit average length, $\mu$ is permeability, and $S$ is magnetic flux area. For single stage coil, the magnetic induction coefficient $\mathrm{K}$ is [9]:

$$
K=\frac{4 \pi^{2} N^{2}\left(r_{3}+r_{2}\right)^{2} K_{n}}{I} \times 10^{-6}
$$

In formula, the $\mathrm{r} 3$ is the piston radius, the $\mathrm{r} 2$ is the groove radius, and the $\mathrm{Kn}$ is the geometric coefficient.

$$
K_{n}=\frac{1}{1+0.9 \frac{r_{3}+r_{2}}{L_{1}-2 L_{2}}+0.32 \frac{r_{3}-r_{2}}{r_{3}+r_{2}}+0.84 \frac{r_{3}-r_{2}}{L_{1}-2 L_{2}}} \text {. }
$$

For the two-stage coil, the magnetic induction coefficient $\mathrm{L}$ is:

$$
L=L_{1}+L_{2}+2 M_{12} \text {. }
$$

In formula, the $\mathrm{L}$ is the total magnetic induction coefficient; L1 and L2 are the magnetic induction coefficients of group 1,2 coils; and M12 is the mutual inductance coefficient.

\section{Magnetic Circuit Structure Optimization}

Response time is an important indicator to measure the performance of MRF damper. Control period, application range, and operation characteristics are directly determined by the response time [10-11]. In order to improve the magnetic circuit performance and shorten the response time, different magnetic circuit structures and key parameters should be considered, and the magnetic circuit optimization design should be combined with the magnetic properties of the material.

Based on the above theoretical analysis, optimization of the key parameters can improve the distribution of the magnetic field.

According to the above theoretical analysis, by optimizing the key parameters, the magnetic field distribution and magnetic circuit performance can be improved, and the response time can be shorten. The optimum design method, based on magnetic circuit can make the MRF damper compacted, adjustable coefficient increased, energy efficiency improved, and response speed accelerated.

From the Eq. (13) analysis, response time is proportional to the ratio of the magnetic induction coefficient to the magnetoresistance K/R. Under the condition that the power consumption of the absorber is not increased, reduce the coefficient $\mathrm{K}$ of magnetic induction can improve the response speed of MRF damper. The power consumption of the damper is I2R, and the reluctance $\mathrm{R}$ remains unchanged, that is, the average length of the magnetic circuit is unchanged by b. Double stage coil structure can be adopted to reduce magnetic induction coefficient K [12]. The equation (16) shows 
that the mutual inductance coefficient M12 in front of the sign depends on each coil winding direction and polarity. Figure 5 is connected as the polarity of the mutual inductance coefficient from a negative coefficient of magnetic induction, magnetic coil $\mathrm{L}$ minimum response time. Figure 6 is the distribution map of the double circuit lines of shock absorber of Fig. 5.

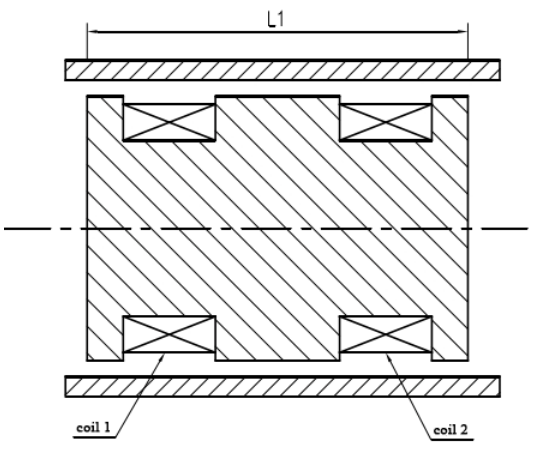

Fig. 5 Structure of double stage MRF damper
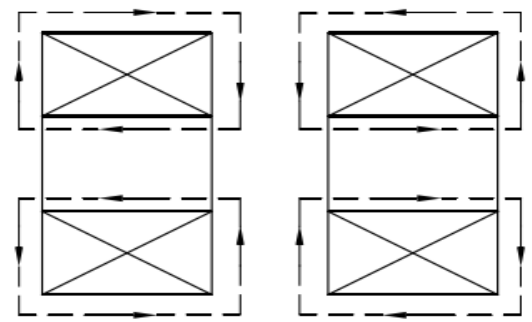

Fig. 6 Distribution of magnetic lines in dual magnetic circuit

The MRF damper designed in this paper uses the double stage magnetic circuit with opposite direction, at the same time increasing the thickness of the working cylinder and the diameter of the piston to improve the magnetic characteristics of the absorber. The specific structural parameters are shown in Table 1.

Table 1 Structural parameters of MRF damper

\begin{tabular}{|c|c|c|c|}
\hline $\begin{array}{c}\text { Structural } \\
\text { parameters }\end{array}$ & $\begin{array}{c}\text { single pole } \\
\text { unoptimized } 1\end{array}$ & $\begin{array}{l}\text { single pole } \\
\text { optimized } 2\end{array}$ & $\begin{array}{l}\text { single pole } \\
\text { optimized } 3\end{array}$ \\
\hline Working gap/mm & 1 & 1 & 1 \\
\hline Piston diameter $/ \mathrm{mm}$ & 25 & 30 & 30 \\
\hline Piston length $/ \mathrm{mm}$ & 50 & 50 & 50 \\
\hline Damping channel length /mm & 11 & 11 & $\begin{array}{c}\text { 7(Both ends) } \\
\text { 8(Middle) }\end{array}$ \\
\hline Working cylinder thickness /mm & 1.5 & 2.5 & 2.5 \\
\hline Piston rod diameter $/ \mathrm{mm}$ & 10 & 10 & 10 \\
\hline Coil groove diameter $/ \mathrm{mm}$ & 15 & 15 & 15 \\
\hline Current strength/A & 1 & 1 & 1 \\
\hline Coil turns/N & 300 & 300 & 300 \\
\hline
\end{tabular}

The structural parameters of the MRF damper are determined by theoretical calculation. The effects of the piston diameter, the thickness of the working cylinder and the multiple magnetic circuits on the response time are discussed. The results show that [14-15], reasonable selection of structural parameters and multistage magnetic circuit can improve the magnetic circuit distribution of the shock absorber, optimize the magnetic field strength, improve the damping force and shorten the response speed of the magnetorheological fluid shock absorber. 


\section{Electromagnetic finite element simulation and analysis}

In this paper, ANSYS finite element software is used to simulate and analyze the electromagnetic field distribution characteristics of MRF damper. By analyzing the simulation results of magnetorheological fluid dampers with different structures, the influence of different structural parameters on the magnetic characteristics of the absorber is discussed. In the design of magnetic circuit, the magnetic leakage effect is neglected and the electromagnetic field distribution of 3 MRF dampers in Table 1 is analyzed by finite element method.Fig.7 9 is a magnetic flux density profile of magnetorheological fluid dampers 1,2 , and 3 .
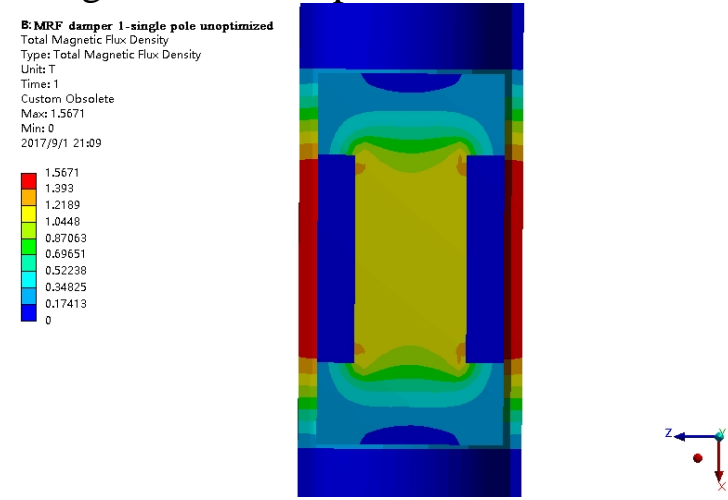

Fig. 7 Magnetic flux density of MRF damper 1

Fig. 7 is a magnetic field distribution diagram of unoptimized MRF damper 1. It can be seen from the figure that the magnetic circuit structure is single-stage coil, and when current is $1.0 \mathrm{~A}$, the range of absorber damper channel flux density is 0 to $0.348 \mathrm{~T}$, and the magnetic field distribution is very uneven. The maximum magnetic flux density of the working cylinder of the magnetic circuit is 1.5671T, which exceeds the saturation magnetic induction intensity of the working cylinder. The magnetic flux density of the magnetic circuit is $1.2189 \mathrm{~T}$, and it also exceeds the saturation magnetic induction intensity of the piston. The magnetic flux density damping channel work requirements should be 0.3-0.6T and the magnetic circuit working cylinder and piston should be in its material saturation magnetic induction density, but magnetic properties of magneto rheological damper 1 failed to meet the requirements of the job.
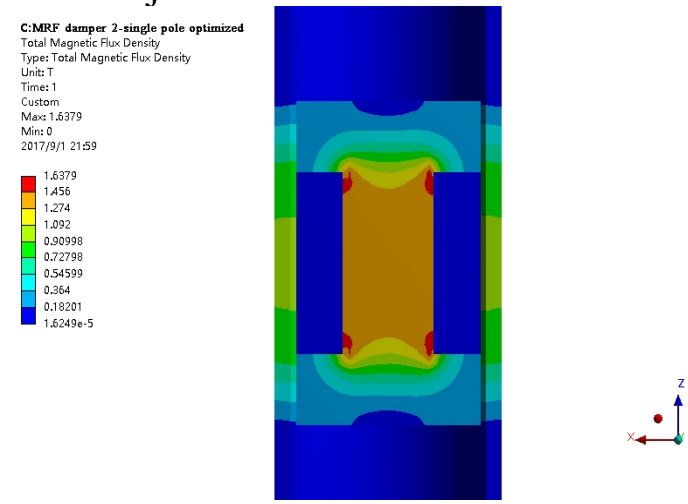

Fig. 8 Magnetic flux density of MRF damper 2

Fig. 8 shows the magnetic field distribution of the optimized MRF damper 2. The MRF damper 2 is optimized for the magnetic circuit structure based on the MRF damper 1 . The piston diameter is extended by $5 \mathrm{~mm}$ and the working cylinder thickness is thicker than $1 \mathrm{~mm}$ and the other structural parameters remain unchanged. Through optimization, the magnetic flux density of the working cylinder is $1.092 \mathrm{~T}$, which has exceeded the saturation magnetic induction intensity of the working cylinder. The magnetic flux density of damper channel is 0.21-0.54T, and the magnetic field distribution is relatively uniform, which is close to the flux density of the work requirement. But in the actual magnetic circuit, the material has been saturated with magnetic flux, and the flux density 
can not be increased. Therefore, the magnetic circuit structure is optimized to improve the distribution of the magnetic field.

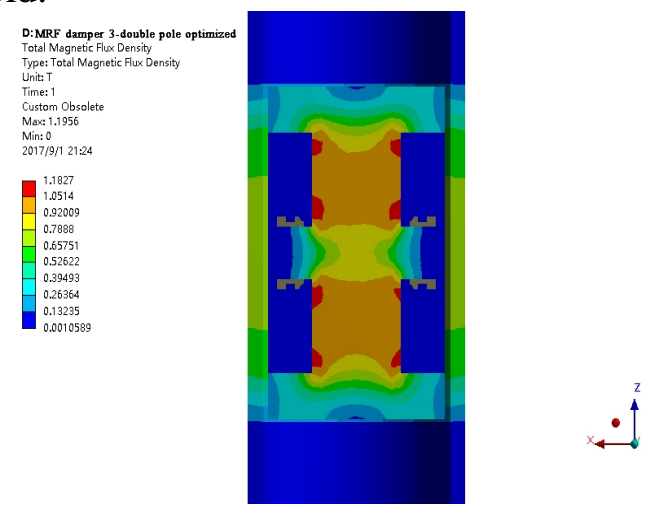

Fig. 9 Magnetic flux density of MRF damper 3

Fig. 9 is a magnetic field distribution diagram of double stage magnetorheological fluid damper 3. The MRF damper 3 has the same configuration parameter as the MRF damper 2, but the coil is divided into two groups, each coil have 150 turns and reverse winding. As can be seen from the diagram, the magnetic field distribution of the shock absorber is obviously improved. The magnetic field distribution of the piston is uniform and the maximum magnetic flux density is $1.1827 \mathrm{~T}$. The magnetic flux density of the working cylinder in the magnetic circuit is $0.7888 \mathrm{~T}$, which is much lower than the saturation magnetic induction intensity of the working cylinder. The magnetic flux density damping channel is from 0.33 to $0.65 \mathrm{~T}$, the magnetic field distribution is even, and magnetic flux density can meet the requirements of magneto rheological damper. Through theoretical analysis, magnetorheological fluid damper 3 can effectively improve circuit performance and reduce the response speed .

\section{Conclusions}

Aiming at the classical magnetic circuit structure of magnetorheological fluid shock absorber, the key parameters and the magnetic circuit structure of the absorber are optimized in order to improve the response speed of the magnetic circuit. Using ANSYS software for simulation analysis, the results of the study can be seen: (1) Single pole not optimized MRF damper 1, the circuit lines are parallel to the working cylinder and perpendicular to the magnetorheological fluid, the magnetic flux density of the working cylinder is the largest, magnetic saturation has occurred. The magnetic circuit performance and response speed of MRF damper are severely impacted. According to the theoretical analysis, the magnetic field distribution can be improved by optimizing the key parameters or adopting multilevel magnetic circuit. (2) The MRF damper 2 changes the cross sectional area of the magnetic flux lines and reduces the magnetic flux density of the working cylinder by increasing the thickness of the working cylinder and the diameter of the piston. After optimization, the magnetic field distribution of the damper is more uniform, but the magnetic saturation of the working cylinder material and the damping channel density cannot meet the requirements of the work. (3) MRF damper 3 magnetic circuit adopts double pole reverse winding way, lines from two independent loops, and its magnetic field distribution is even. If the excitation magnetic field is superposed on the damping channel, the magnetic circuit utilization ration can be improved, the magnetic flux density of the damping channel is reduced, and the response speed of the shock absoeber is improved. Compared the optimized two-stage magnetorheological fluid damper with single stage magnetorheological fluid damper, the optimized two-stage magnetorheological fluid damper can effectively shorten the response time, the magnetic performance is more superior, the magnetic field distribution is more reasonable, so as to verify the validity and correctness of the magnetic circuit structure method. 


\section{References}

[1] H. H. Zhang, C. G. Liao, and W. M. Chen,. J. Funct. Mater. Devic. Vol. 10(4), (2004), p. 493497.

[2] DENG Xiaoyi, SHI Mingquan, FAN Shuqian. COMPUT S Vol. 27(9),( 2010), p. 291-294.

[3] CHEN Kunshan, YUAN Shanfa, and LIU Shaoqing. Trac. F. Trans. Vol. 34(4), ( 2007), p. 7981.

[4] M.R.Jojjy, J.W.Bender and J.D.Carlson. SPIE 5th Annual Int. Symposium on Smart Structures and Materials, San Diego, CA, Vol. 15, (1998).

[5] ZHU Shixing, and LU Mingtao. Mach. Des. Manu. Vol. (3), (2013), p. 41-45.

[6] ZHENG Ling, LI Yinong, and HU Yong. J. Vibra. Eng. Vol. 21(2), (2008), p. 173-178.

[7] LIAO Changrong.Research on magnetorheological damper of automobile suspension system[D]. Chongqing University, (2001).

[8] LIAO Changrong, YU Miao, and CHEN Weimin. China Mech. Eng. Vol. 13(16), (2002), p. 1391-1394..

[9]Welsby V G. The Theory and Design of Inductance Coils[J]. Radio Engineers Journal of the British Institution of. Vol. 11(1),(1960)

[10] GUAN Xinchun, and OU Jinping. Earth. Ingin. Eng. Vibra Vol. 22(6), (2002), p. 96 -102.

[11] ZHANG Lin. Machinery. Vol. 46(531), (2008), p. 37-38.

[12] Lan Wenkui, Zheng Ling, and Li Yinong. Trans. Chinese Soc. Agi. Machi. Vol. (4), (2007), p. 142-146.

[13] LanWenkui. Experimental Study and Design of MR Damper[D]. Chongqing University, 2007.

[14] Anderson E H. Smart Structures and Materials 2003: Industrial and Commercial Applications of Smart Structures Technologies[J]. Proceedings of SPIE - The International Society for Optical Engineering, 2003, 5054(3):259-268.

[15] Choi S B, Lee S K, and Park Y P. J. S. Vibra. Vol. (245), (2000), p. 375-382. 